\title{
EL USO DE AUNQUE EN EL ESPAÑOL PENINSULAR: UN ANÁLISIS DISCURSIVO- FUNCIONAL
}

\author{
THE USE OF AUNQUE IN PENINSULAR SPANISH: A DISCURSIVE-FUNCTIONAL \\ ANALYSIS
}

\author{
Hella Olbertz \\ Universiteit van Amsterdam \\ h.g.olbertz@uva.nl \\ Talita Storti Garcia \\ Universidade Estadual Paulista \\ talita@ibilce.unesp.br \\ Beatriz Goaveia Garcia Parra \\ Universidade Estadual Paulista \\ biagarcia.parra@hotmail.com
}

En este trabajo describimos los usos de aunque en un corpus de obras literarias publicadas en España. Nos valemos de la Gramática Discursivo-Funcional como instrumento descriptivo aunque sin entrar en detalles del formalismo correspondiente. La conjunción aunque se usa en contextos concesivos y concesivo-condicionales, que de modo general se distinguen por el uso del modo indicativo en la prótasis concesiva y del subjuntivo en la concesivo-condicional. En las oraciones concesivas aunque introduce modificadores del enunciado o de la enunciación. En el primer caso, la cláusula concesiva actúa como modificador adverbial de apódosis eventivas o proposicionales, mientras que en el segundo, modifica actos discursivos o entidades comunicativas mayores, los así llamados movimientos. En los contextos concesivo-condicionales la prótasis introducida por aunque es de naturaleza escalar y puede expresar condiciones reales o irreales. Aparte de modificar apódosis eventivas y proposicionales, la cláusula concesivo-condicional puede expresar propiedades hipotéticas mínimas de eventos.

Palabras clave: conjunción aunque, oraciones concesivas, oraciones concesivo-condicionales, Gramática Discursivo-Funcional

Keywords: conjunction aunque, concessive sentences, concessive conditional sentences, Functional Discourse Grammar

In this study we describe the use of aunque in Spanish narrative texts. We use Functional Discourse Grammar as a descriptive tool, without however going into technical details. The conjunction aunque is used for the expression of concessive and concessive conditional meanings, which can, globally, be distinguished through the use of the indicative in the former and that of the subjunctive in the latter case. In concessive sentences, aunque can modify descriptive or interactional apodoses. In the first case, the concessive clause acts as an adverbial modifier of either events or propositions, 
whereas in the second, the concessive clause modifies either a discourse act or a larger communicative entity, the so-called move. In conditional concessive contexts, the aunque protasis is of a scalar nature and can express real or unreal conditions. In addition to modifying eventive and propositional apodoses, the concessive conditional clause can express hypothetical minimal properties of events.

(Recibido: 20/01/2016; Aceptado: 13/06/2016)

\section{Introducción ${ }^{1}$}

Como se sabe de las muchas publicaciones sobre la semántica concesiva (p.ej. König 1985, 1988, 1994; Crevels 1998, 2000; Garachana Camarero 1999; Flamenco García 1999; Neves 1999), se trata de una relación contrastiva en la cual lo afirmado en la subordinada concesiva no concuerda con lo que se esperaría dado el contenido de la cláusula ${ }^{2}$ principal. Considérese el siguiente ejemplo citado de la Nueva gramática de la lengua española (RAE 2009: 3599):

1. Aunque estaba muy cansada por el viaje, impartió una conferencia magnífica.

Bajo circunstancias normales, no se esperaría que una persona muy cansada fuera capaz de presentar algo magnífico. Generalizando un poco más, se puede decir que se produce un evento (o “estado de cosas") $q$ pese a la presuposición general de que dada una situación $p$, se excluye la realización de un estado de cosas $q$. La relación entre la oración concesiva y esta presuposición o background assumption (König 1988: 147) se puede formalizar así:

2. 'aunque $p, q$ ' presupone 'si $p$ normalmente $\sim q$ '

Esta representación ya indica que existe una relación semántica entre las oraciones concesivas y las condicionales. En la Nueva gramática de la lengua española esta relación se describe diciendo que "entre los componentes de los períodos concesivo y condicional se establece una relación de interdependencia en el sentido de que ninguno de los dos miembros puede ser suprimido sin alterar el significado del conjunto, o incluso comprometer la gramaticalidad de la construcción" (RAE 2009: 3530). Dada esta analogía, usaremos los términos "prótasis" para la cláusula concesiva y “apódosis" para la cláusula principal (König 1988; Flamenco García 1999; Neves 1999; RAE 2009).

El presente estudio versa sobre el uso de la conjunción concesiva aunque, que no es sólo la conjunción concesiva más frecuente en español sino también la más flexible en términos semánticos. Trataremos exclusivamente el uso de esta conjunción en contextos de cláusulas con verbo finito como en los siguientes ejemplos:

3. En cuanto a mí, aunque mi nombre es Marcos, Don Fortunato me llamaba Zaratustra. (Merino)

4. [Monólogo interior del protagonista, lleno de odio de su interlocutor] Julio no le engañaría, aunque lo intentase. (Pombo)

\footnotetext{
${ }^{1}$ Agradecemos a Magaly Grández Ávila, a Kees Hengeveld y a una revisora anónima por sus útiles comentarios a versiones anteriores de este artículo. Los errores que quedan son nuestros. Parte de este trabajo ha sido financiado por medio de la beca no. 2015-00035-4 de la Fundação de Amparo à Pesquisa do Estado de São Paulo (FAPESP).

2 Para evitar confusiones nos valemos del término cláusula para referirnos a una oración parcial, subordinada o principal, reservando el término oración para la combinación de las cláusulas en un período.
} 
En (3), aunque se usa con el verbo de la prótasis en indicativo, mientras que en (4), el verbo tiene una forma de subjuntivo. El uso del subjuntivo en el ejemplo (4) se explica por el carácter hipotético del contenido de la prótasis. La oración concesiva hipotética ejemplificada aquí se suele denominar concessive conditional en inglés (e.g. König 1985; Haspelmath y König 1998), concepto que, siguiendo a Flamenco García (1999), traduciremos como 'concesivo-condicional'. La diferencia entre las oraciones concesivas propias y las concesivo-condicionales es el primer problema que trataremos en detalle en el presente trabajo.

El segundo problema que pretendemos analizar es el tipo de entidad semántica o pragmática representada por la oración concesiva y la concesivo-condicional, tal como se presenta en Sweetser (1990), aplicado por Crevels $(1998,2000)$ a la concesión. Para explicar este problema, nos limitaremos aquí a los casos concesivos. Considérense los siguientes ejemplos:

5. En ocasiones, Mucius Scaevola emprendía un solo de violín, aunque no era infrecuente que accediese a acompañarles en la interpretación de las más famosas melodías de las operetas de moda. (García Hortelano)

6. Y aunque la soledad obsesiva de tantos años le había dado al recuerdo del suceso una comezón cainita, lo cierto era que su remordimiento primero se originó, sobre todo, más que en aquella muerte, en la conciencia de sus resultados. (Merino)

En el ejemplo (5), la prótasis modifica un evento, y como tal es ilustrativo del "nivel de contenido" (content level) de Sweetser (1990: 78-79), que equivale al "estrato de estado-de-cosas" (layer of the state-of-affairs) de Hengeveld y Mackenzie (2008: 166-181). La situación es diferente en (6), en la que la prótasis no modifica un evento, sino un contenido proposicional (Hengeveld y Mackenzie 2008: 144-157). En la clasificación de Sweetser (1990: 78-79), el ejemplo (6) corresponde al "nivel epistémico", por estar relacionado con el conocimiento del hablante o narrador. Las prótasis concesivas (5) y (6) tienen la propiedad común de concernir al enunciado, es decir, al contenido descriptivo de la apódosis, así que denominaremos este tipo de construcción "concesiva descriptiva".

La relación entre la prótasis y la apódosis es esencialmente diferente en el caso de (7), porque en este caso la apódosis representa un acto ilocutivo, mientras que la función de la prótasis sirve como atenuación retórica, indicando que el/la hablante está consciente de la infelicidad de este acto dentro de la interacción verbal específica (y, por lo tanto, de lo que "normalmente" debería impedir su realización).

7. ¿Te has reconciliado con Roberto?, aunque ya adivino la respuesta. (adaptado de RAE 2009: 3605)

Desde el punto de vista sintáctico, cabe observar que en (7), la prótasis adquiere independencia sintáctica de la apódosis, formando una cláusula independiente, que sigue siendo, sin embargo, interaccionalmente dependiente de la apódosis (Hengeveld y Mackenzie 2008: 54-56). Para referirse al tipo de concesivas ilustradas en (7), Crevels (1998) hace uso del término "concesiva ilocutiva" (véase también RAE 2009: 3604-3606), que la autora distingue de la "concesiva textual", que tiene un alcance más amplio (Crevels 1998: 137-139). Tanto las concesivas ilocutivas como las textuales tienen la propiedad de aludir a la enunciación, es decir, a las estrategias del hablante en la interacción verbal. Nos vamos a referir a las concesivas de enunciación con la etiqueta de "concesiva interpersonal" siguiendo a Hengeveld y Mackenzie (2008: cap. 2).

El marco teórico del cual nos valdremos en este trabajo es la gramática discursivo-funcional (GDF) (Hengeveld y Mackenzie 2008), aunque, por razones de espacio, no vamos a entrar en detalles sobre el formalismo de la teoría. 
El modelo de la GDF nos ayudará a aclarar de una manera sistemática (i) la diferencia entre las construcciones concesivas y concesivo-condicionales y (ii) la semántica y pragmática de los usos descriptivos e interpersonales de las dos.

El estudio presentado aquí se basa en primer lugar en un corpus literario de obras publicadas en España entre los años 1970 y 1990 (101.532 palabras), que rindió 115 casos de aunque, de los cuales 91 introducen prótasis finitas. Se eligió un corpus cerrado para llegar a tener una visión general de la variación que existe en el campo de la concesión. Además citaremos otros ejemplos de diversas fuentes de datos auténticos del español peninsular.

Este artículo está organizado de la siguiente manera: la sección 2 está dedicada a la diferencia entre las oraciones concesivas y las concesivo-condicionales; en la sección 3, trataremos las oraciones concesivas, ocupándonos primero de algunas propiedades generales de la concesión para pasar después a sus usos descriptivos e interpersonales; en la sección 4, discutiremos las construcciones concesivo-condicionales y en la sección 5, finalmente, presentaremos unas breves conclusiones.

\section{Oraciones concesivas y oraciones concesivo-condicionales}

La mejor manera de ilustrar la diferencia entre la concesiva propia y la concesivo-condicional es considerar los pares mínimos. Considérese el ejemplo concesivo-condicional en (8a) y su variante concesiva en $(8 b)$ :

8. a. EL PAÍS ha cumplido con rigor el compromiso con sus lectores: publicar información veraz y de relevancia para la opinión pública, aunque ello incomode a los poderes establecidos. (El País, 10/03/2013).

b. [...] aunque ello incomoda a los poderes establecidos.

Existe una clara diferencia semántica entre el original en (8a) y su variante en (8b): mientras que en la concesivo-condicional del original, la redacción del periódico se compromete a los principios de libertad e independencia de prensa, que tiene en cuenta la posibilidad hipotética de ser molesto para el gobierno, en la concesiva en (8b) se hace referencia al caso concreto y factual de ser molesto para el gobierno. Es decir que la combinación de aunque con el verbo en subjuntivo en (8a) expresa un caso hipotético, mientras que la combinación de aunque con el verbo en indicativo en (8b) expresa una situación factual. El carácter hipotético de (8a) indica la relación que existe entre las cláusulas concesivo-condicionales y las condicionales, que también expresan hipótesis. Los siguientes ejemplos, adaptados de Bülow y Schamberger (2013: 290), pueden servir para aclarar esta relación:

9. a. Si usted da una charla en el congreso no voy a participar. b. Aunque usted dé una charla en el congreso no voy a participar. c. Aunque usted da una charla en el congreso no voy a participar.

En el ejemplo (9a), que, por cierto, es bastante ofensivo, el valor de verdad en la prótasis se marca como indeterminado por la partícula hipotética si, lo cual tiene el efecto de suspender automáticamente el valor de verdad de la apódosis; es decir que ambas son no-factuales. En (9b), el efecto combinado de la conjunción concesiva y el subjuntivo marcan la prótasis como no factual, sin que esto afecte a la apódosis, que sigue siendo factual. En (9c), finalmente, tanto la prótasis como la apódosis son factuales (Flamenco García 1999: 3823). Con respecto al contenido de las oraciones en su totalidad, estas combinaciones tienen los siguientes efectos: la de (9a) no es factual, la de (9c) es factual y la de (9b) es parcialmente factual, i.e. la apódosis es real o verdadera (Haspelmath y König 572-573), mientras que la prótasis es hipotética. 
Por lo tanto, las oraciones concesivo-condicionales se llaman también "semi-factuales" o "concesivas hipotéticas"; también se suele usar la etiqueta "condicionales de irrelevancia", porque el carácter hipotético de la prótasis es irrelevante para la factualidad de la apódosis (Haspelmath y König 1998: 563). Nosotras nos atenemos al concepto de "concesivo-condicional" por ser el más común en la literatura.

Finalmente, es importante darse cuenta de que existen varios tipos de concesivocondicionales $^{3}$, y que el tipo expresado por medio de aunque pertenece a las así llamadas concesivo-condicionales escalares, porque en la prótasis se expresa un valor extremo en una escala imaginaria de valores posibles en un contexto dado (Flamenco García 1999: 3843-3844). Otra manera de expresar la relación concesivo-condicional escalar es a través de incluso si, tratado en detalle por Rodríguez Rosique (2012: 106-108), quien da los siguientes ejemplos:

10. a. Parece ser que tomar un suplemento de ácido fólico antes del embarazo es bastante preventivo e incluso si se toma después de haber detectado espina bífida en el feto, reduce la gravedad de la lesión. (El Mundo, suplemento 'Salud', 01/05/1997, CREA, cit. por Rodríguez Rosique 2012: 106)

b. [...] y si se toma incluso después de haber detectado espina bífida en el feto, reduce la gravedad de la lesión. c. [...] aunque se tome después de haber detectado espina bífida en el feto, reduce la gravedad de la lesión.

La diferencia esencial entre las variantes con incluso sí (10a y 10b) y con aunque (10c) es el grado más alto de gramaticalización de la expresión en (10c): como se puede ver en (10b), la partícula incluso no necesita preceder a la conjunción si, sino que puede ocupar varios lugares dentro de la prótasis, mientras que aun y que en la conjunción aunque están fusionados.

Habiendo aclarado la diferencia entre los usos concesivos y concesivo-condicionales con aunque, pasaremos ahora a la discusión de las propiedades semánticas, pragmáticas y gramaticales de cada uno individualmente, comenzando con el uso concesivo.

\section{Oraciones concesivas con aunque}

En esta sección trataremos tres cuestiones, siendo las primeras dos de índole más general. Empezamos con la relación que existe entre la concesión y la adversatividad en 3.1, pasando luego a la diferencia entre el uso del indicativo y el subjuntivo en las cláusulas concesivas en 3.2. La sección 3.3. trata uno de nuestros temas centrales, el de los usos descriptivos e interpersonales de aunque. Terminaremos con un resumen en 3.4.

\subsection{Concesión y adversatividad}

Las oraciones concesivas y las adversativas comparten la propiedad de expresar una relación de contraste entre sus partes componentes, y por esto en muchos casos, es posible sustituir, mutatis mutandis, la oración concesiva por una adversativa, sin que haya cambio de sentido. Un caso ilustrativo es el ejemplo (1), que repetimos aquí como (11a), y su variante adversativa en (11b):

11. a. Aunque estaba muy cansada por el viaje, impartió una conferencia magnífica.

b. Estaba muy cansada por el viaje, pero impartió una conferencia magnífica.

\footnotetext{
${ }^{3}$ Los otros tipos concesivo-condicionales, son los alternativos (i) y los de cuantificación universal (ii):

i. al estar España metida en esa:/ en la Otan/ macho// quiera o no quiera/ la iban a obligar/ (Moreno Fernández et al. 2004: 71)

ii. y no logrará comprender jamás por más vueltas que le dé (Tusquets)

Véanse Flamenco García (1999: 3846-3849) y RAE (2009: 3616-3621) para la expresión de estos tipos en español, y Haspelmath y König (1998) para el análisis de las expresiones españolas desde una perspectiva tipológica.
} 
A pesar de que los límites entre la concesión y la adversatividad son crónicamente difíciles de identificar, existen casos concesivos "que no admiten de manera tan evidente [como en nuestro ejemplo (11)] la sustitución por pero sin que el sentido se vea afectado" (RAE 2009: 3605):

12. Saludando fugazmente a algunos conocidos, Biralbo vino hacia mí, aunque en ningún momento había dado señales de verme mientras tocaba (A. Muñoz Molina, El invierno de Lisboa, cit. por RAE 2009: 3605).

König (1985: 5) demuestra que una característica de la relación adversativa es que puede darse entre estados de cosas (en adelante EEdCC) o proposiciones que al parecer no tienen relación directa entre sí (véase también Flamenco García 1999: 3813). En nuestro corpus encontramos el siguiente ejemplo:

\footnotetext{
13. [Carta escrita por un estudiante a un amigo, durante la Guerra Civil española] a. Pensé en darle sepultura, pero prohibieron el paso en la zona. (Gironella)

b. ?"Pensé en darle sepultura, aunque prohibieron el paso en la zona.

c. ??Prohibieron el paso en la zona, aunque pensé en darle sepultura.
}

Para explicar este caso, vale la pena reconsiderar primero la estructura semántica de la concesión. Hemos visto que la relación concesiva se correponde con una situación en la cual existe una presuposición de que, dada la proposición o el evento p,queda excluida la verdad de la proposición o la realización del evento q. König (1985: 4) expresa esta presuposición por medio de la siguiente fórmula informal:

\section{Normalmente (si $p$, entonces no- $q$ )}

Obviamente no se puede aplicar la fórmula en (14) al ejemplo (13a), ya que ninguna de las dos reformulaciones en términos concesivos es plausible; (13b) es extraño porque el hecho de la prohibición del paso fúnebre no puede ser un impedimento para concebir la idea de dar sepultura a una persona querida, y (13c) es aun más problemático porque expresa que la idea de dar sepultura es impedimento para la prohibición del paso ${ }^{4}$. Aparte de esto, es el tiempo de pasado perfectivo en la oración adversativa que hace que sean extrañas las reformulaciones concesivas, ya que en la cláusula concesiva sería normal el pasado imperfectivo.

Los ejemplos (12) y (13) demuestran que, pese a sus similitudes, los conceptos de adversatividad y concesividad son distintos. König (1985) comenta que las conjunciones adversativas en vez de establecer una relación de incompatibilidad entre las dos cláusulas, 'conectan dos proposiciones que apoyan conclusiones contradictorias, ${ }^{5}$. Basándose en Anscombre y Ducrot (1977), König continúa explicando que "una oración en la forma " $p$ pero q" expresa que la primera cláusula " $p$ " es un argumento a favor de una conclusión " $r$ ", mientras que la segunda cláusula " $q$ " apoya la conclusión exactamente opuesta, "no-r", y esta última conclusión es la que tiene más peso' (König 1985: 6) ${ }^{6}$, siendo por tanto la decisiva. La siguiente fórmula, citada de Anscombre y Ducrot (1977: 28), resume esta relación semántica, donde la flecha simboliza "conclusión":

$$
\begin{aligned}
& \text { 15. } p \text { pero } q \\
& \text { a. } p \rightarrow r \\
& \text { b. } q \rightarrow \text { no- } r \\
& \text { c. } q \text { lleva más peso }
\end{aligned}
$$

\footnotetext{
${ }^{4}$ Flamenco García (1999: 3818) explica que, en general, son incompatibles con la concesión las expresiones de volición y obligación.

5 "What adversative conjunctions like but do is to relate two propositions that support contradictory conclusions."

6 "a sentence in the form ' $p$ but q' expresses that the first clause ' $p$ ' is an argument for a conclusion ' $r$ ', whereas the second clause ' $q$ ' supports the very opposite conclusion 'not $r$ ' and this second conclusion carries more weight in the whole argument."
} 
Es decir que la relación adversativa puede existir entre dos proposiciones que no están relacionadas inmediatamente entre sí, porque lo que está relacionado son sólo las conclusiones. Aplicando esta fórmula al ejemplo en (13a), el hablante quiere dar sepultura a su ser querido ( $p$ ), la conclusión $(r)$ es que ello implica paso fúnebre, sin embargo, el paso fúnebre está prohibido $(q)$, lo cual lleva a la conclusión de que (no-r), es decir que no va a haber paso fúnebre.

La concesiva se distingue de la adversativa por el hecho de que los contenidos de la apódosis y la prótasis siempre están estrechamente relacionados, ya que la unión de estas dos cláusulas se opone a la relación causal presupuesta. La fórmula en (14), expresa precisamente eso: la existencia de una relación causal esperada entre $p$ y no- $q$. En (12a), por ejemplo, se esperaría que la aparente falta de interés del músico para con el narrador interno causase un desencuentro entre los dos personajes, mientras que lo que se describe en la apódosis es precisamente lo contrario, un encuentro particularmente amistoso. Para definir la relación semántica entre la causalidad y la concesión en la oración concesiva, König (1994: 680) propone etiquetas como "anticausa", “incausal” o "causa inoperante" (véase también Flamenco García 1999: 3813).

No obstante las diferencias entre la concesión y la adversatividad, es importante recordar lo que tienen en común: las dos comparten la propiedad de expresar una relación de contraste (König 1985: 6). Debido a esta proximidad semántica, la conjunción concesiva se usa a veces en función adversativa:

16. a. Yo era una turista más, ni siquiera sabía si era americana, inglesa o española. Aunque lo acabó sabiendo. (Puértolas) b. Yo era una turista más, ni siquiera sabía si era americana, inglesa o española. Pero lo acabó sabiendo.

17. a. Netanyahu tiene todos las cartas para repetir como primer ministro, aunque debe entender el mensaje: la sociedad israelí no quiere más de lo mismo, pide renovación de políticos. (El País 25/01/2013)

b. Netanyahu tiene todos las cartas para repetir como primer ministro, pero debe entender el mensaje: la sociedad israelí no quiere más de lo mismo, pide renovación de políticos.

En estos dos casos no hay prótasis ni apódosis sino una coordinación adversativa de dos oraciones autónomas. Este fenómeno no es específico del español sino que se suele dar de modo general en las lenguas que poseen expresiones específicas concesivas (König 1985: 7) ${ }^{7}$.

\subsection{El uso del subjuntivo en las prótasis concesivas}

En la subordinación adverbial concesiva propia, el uso del subjuntivo generalmente tiene la función de marcar el conocimiento que comparten el hablante / escritor y el oyente / lector (Flamenco García 3829-3830) ${ }^{8}$. Considérense los siguientes ejemplos:

18. [En la Guerra Civil] Gildo y Juan, envueltos en sus capotes, duermen cerca de nosotros, apenas dos bultos negros en la oscuridad. (...) Juan no ha cumplido los dieciocho años todavía y Gildo tiene más de treinta. Casi podrían ser padre e hijo, aunque ahora duerman hombro con hombro, amenazados por un mismo temor. (Llamazares)

19. Don Fortunato señaló el anillo: - Siempre quise comprarle ese anillo. Ya sabe usted que empleo parte de mi tiempo y de mis modestos medios en cuidar y ampliar mi colección de anillos. Dada la rareza de la figura, y aunque el anillo en sí no sea muy rico, le puedo hacer una buena oferta. (Merino)

\footnotetext{
${ }^{7}$ Desde una perspectiva tipológica König (1994: 179) demuestra que la expresión gramatical de la concesión es de origen relativamente reciente, en las lenguas que la poseen, pues hay muchas lenguas en el mundo que se valen exclusivamente de expresiones adversativas para expresar la relación de contraste (König 1988: 149). Por lo tanto, se puede sostener que la adversidad es un concepto semántico más básico (König 1985: 7).

${ }^{8}$ Nótese que el subjuntivo en la prótasis concesiva puede tener funciones no relacionadas con la concesión, como por ejemplo la de expresar el bajo grado de compromiso del hablante con la veracidad del contenido proposicional:

(i) - ¿Quién dice que los monos no tienen cola? [...]

-En efecto, la tienen-intervino el dominico joven-, aunque tal vez no pueda decirse que sea demasiado larga. (Tomeo)
} 
En (18), se habla de los dos hombres que están durmiendo en el contexto inmediatamente precedente a la oración concesiva, y, por tanto, el evento descrito en la prótasis concesiva es conocido. En (19), se habla de las propiedades de un anillo que los participantes en el diálogo narrado pueden ver, es decir que aquí son factores situacionales a los que se debe el conocimiento de los interactantes.

Un caso específico del mismo fenómeno es el llamado 'subjuntivo polémico', donde la cláusula concesiva retoma el argumento del interlocutor para desestimarlo (Flamenco García 1999: 3829; RAE 2009: 3608-3609). En nuestro corpus se encuentra el siguiente ejemplo:

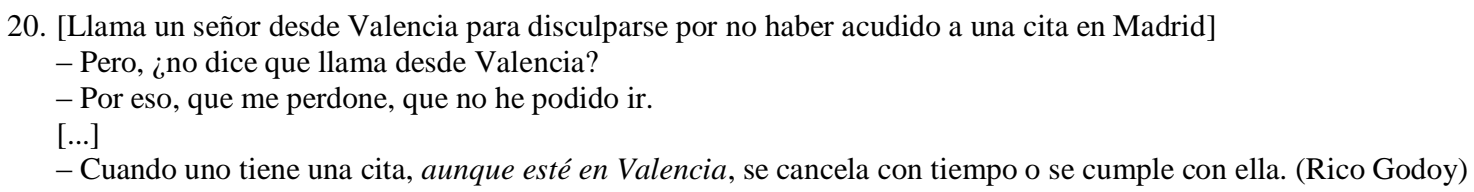

En este ejemplo el señor motiva su falta de acudir a la cita en Madrid por estar en Valencia, motivación que su interlocutora refuta. Lo que tiene en común (20) con los dos ejemplos anteriores es que la cláusula concesiva contiene información conocida por ambos participantes en la interacción.

Una explicación del fenómeno ilustrado en (18)-(20) se nos ofrece en la gramática discursivofuncional, que forma parte de un modelo general de interacción verbal. Dentro de este modelo la diferencia entre la información conocida y la información nueva se explica por la interacción de la gramática propiamente dicha con el así llamado "componente contextual", que almacena todas las informaciones contextuales y situacionales que codeterminan el contenido y la forma de las contribuciones a la interacción (Hengeveld y Mackenzie 2008: 9-12) ${ }^{9}$. En el caso de los ejemplos (18) y (20), se hace uso del contexto inmediatamente precedente, y en el de (19), de la información situacional, que es tal que los participantes están mirando el anillo en cuestión.

Veamos ahora algunos ejemplos con la prótasis concesiva en indicativo:

21. [En una oficina, inmediatamente después de una pelea violenta con un colega]

Silencio. No está el horno para bollos; Chema finge abstraerse en su trabajo aunque los papeles le bailan y no consigue centrar su atención en este expediente de reforma de una planta de prevaporización sistema Karffel. (Palomino)

22. [De noche, la narradora está esperando a su marido]

Voy a mi dormitorio y abro la cama. Aunque me molestan los pantalones, el sostén y el jersey no quiero ponerme el pijama porque no deseo que Antonio llegue y no me vea vestida de calle. (Rico-Godoy)

En los dos casos, el contenido de la prótasis es información nueva. En términos discursivofuncionales esto significa que el componente contextual no contiene datos correspondientes al contenido de las apódosis.

Habiendo explicado las propiedades generales de las oraciones concesivas, pasemos ahora a los niveles semánticos e interpersonales de las apódosis modificadas por las prótasis concesivas.

3.3. Prótasis concesivas descriptivas e interpersonales

\footnotetext{
9 "El componente contextual contiene una descripción de la forma y del contenido del discurso precedente, del entorno perceptible en el que tiene lugar el evento discursivo y de las relaciones sociales entre los participantes. Este tipo de información es relevante para muchos procesos gramaticales, tales como el encadenamiento narrativo, la reflexividad y la voz pasiva" (Hengeveld y Mackenzie 2011: 7).
} 
En la introducción a este trabajo mencionamos la diferencia entre las cláusulas concesivas que sirven para modificar el enunciado, llamadas aquí "concesivas descriptivas", y las prótasis concesivas que modifican la enunciación, a las que llamamos aquí "concesivas interpersonales". En esta sección discutiremos primero aquellas (3.3.1.) y después éstas (3.3.2.).

\subsubsection{Las concesivas descriptivas}

Las concesivas descriptivas son casos de modificación adverbial propiamente dicha, porque la cláusula concesiva no puede ocurrir independientemente. En los ejemplos (23)-(25), se modifican EEdCC por medio de las cláusulas adverbiales concesivas:

23. En cuanto a mí, aunque mi nombre es Marcos, Don Fortunato me llamaba Zaratustra. (Merino)

24. [En el contexto de una descripción del malestar del narrador interno debido a su amor no correspondido] Por todo lo cual, aunque logré aprobar los exámenes de COU en setiembre de aquel mismo año aciago y me matriculé en la Facultad de Derecho, apenas asistía a las clases y no estudiaba nada, de modo que tardé tres cursos en aprobar el primero. (Soriano)

25. Aunque el recuerdo del conflicto le pasa por el ánimo como papel de lija, Chema ha contestado con templanza, casi amablemente. (Palomino)

En cambio, en los ejemplos (26)-(28) las prótasis con aunque sirven para modificar contenidos proposicionales, lo cual es evidente por los subordinadores epistémicos supongo en (26) y lo cierto era que en (28):

26. Supongo que bromeas al hablar como lo haces de Protto Andretti, aunque en tus palabras subyace un matiz de reproche (Delibes)

27. Chema no sabe quién es el patoso, prefiere ignorarlo, es un amigo aunque le haya irritado con esa broma tan facilona. (Palomino)

28. Y aunque la soledad obsesiva de tantos años le había dado al recuerdo del suceso una comenzón cainita, lo cierto era que su remordimiento primero se originó, sobre todo, más que en aquella muerte, en la conciencia de sus resultados. (Merino)

La diferencia entre los EEdCC y los contenidos proposicionales está en que aquéllos se pueden observar y localizar en el tiempo y en el espacio, mientras que éstos no tienen existencia en el mundo y, por lo tanto, no son localizables ni en el tiempo ni en el espacio, sino que sólo se pueden evaluar con respecto a su valor de verdad (Vendler 1967; Lyons 1977: 438-445). La relación entre los dos es que los contenidos proposicionales pueden contener EEdCC, pero no al revés. La manera de comprobar la diferencia entre los EEdCC y los contenidos proposicionales está directamente relacionada con estas diferencias semánticas: en el caso de los contenidos proposicionales debe ser posible la inserción de una expresión de duda, de veracidad o de conocimiento. Para garantizar la comparabilidad de los ejemplos, elegimos insertar los adverbios probablemente o seguramente en la apódosis, dependiendo de cuál cabe mejor en el contexto. La aplicación de esta prueba rinde resultados semánticamente coherentes en (26)-(28), aunque sean redundantes en los casos de (26a) y (28a):

26. a. Supongo que probablemente bromeas al hablar como lo haces de Protto Andretti, aunque en tus palabras subyace un matiz de reproche

27. a. Chema no sabe quién es el patoso, prefiere ignorarlo, seguramente es un amigo aunque le haya irritado con esa broma tan facilona.

28. a. Y aunque la soledad obsesiva de tantos años le había dado al recuerdo del suceso una comenzón cainita, lo cierto era que su remordimiento primero seguramente se originó, sobre todo, más que en aquella muerte, en la conciencia de sus resultados. 
En los ejemplos (23)-(25), en los que aunque modifica un EdC, la aplicación de la misma prueba da resultados semánticamente dudosos:

23. a. ?"En cuanto a mí, aunque mi nombre es Marcos, Don Fortunato seguramente me llamaba Zaratustra.

24. a. ??Por todo lo cual, aunque logré aprobar los exámenes de COU en setiembre de aquel mismo año aciago y me matriculé en la Facultad de Derecho, seguramente apenas asistía a las clases y no estudiaba nada, de modo que tardé tres cursos en aprobar el primero.

25. a. "?Aunque el recuerdo del conflicto le pasa por el ánimo como papel de lija, Chema seguramente ha contestado con templanza, casi amablemente.

Aunque ninguno de los tres ejemplos modificados es realmente agramatical, la inserción de seguramente rinde oraciones bastante extrañas.

La distinción entre EEdCC y contenidos proposicionales que hacemos aquí está relacionada exclusivamente con la semántica de la cláusula modificada, o sea de la apódosis. Sin embargo, Crevels (1998: 136, 2000: 31) sigue una estrategia distinta: la de aplicar nuestra prueba del adverbio epistémico a la prótasis. Tal procedimiento no es del todo confiable porque la semántica de la apódosis es esencialmente independiente de la de la prótasis, es decir que (i) una prótasis proposicional puede modificar un EdC expresado en la apódosis, y (ii) una prótasis eventiva puede modificar un contenido proposicional en la apódosis. El ejemplo (25) es ilustrativo del primero de los dos casos. Ya vimos en (25a) que la apódosis es un EdC, ya que no admite la modificación por un adverbio epistémico. Sin embargo, la prótasis sí es compatible con tal adverbio, y por lo tal la prótasis se clasificará como contenido proposicional:

25. b. Aunque probablemente el recuerdo del conflicto le pasa por el ánimo como papel de lija, Chema ha contestado con templanza, casi amablemente.

Si bien el nivel semántico de la prótasis y de la apódosis coincide en muchos casos, la única prueba confiable del alcance de la cláusula concesiva consiste en la determinación de la semántica de la cláusula principal.

Antes de pasar a describir la estructura semántica de las oraciones concesivas descriptivas desde un punto de vista discursivo-funcional, cabe observar que nuestros datos no confirman las hipótesis de Crevels (1998: 135-136; 2000: 31-32) sobre la naturaleza de las concesivas de EEdCC. Según esta autora, las concesivas modificadoras de EEdCC en español siempre son "restrictivas", término acuñado por Sweetser (1990: 82) en analogía a las cláusulas relativas, o sea que no se separan por una coma de la apódosis ${ }^{10}$. Partiendo de esta idea, Crevels (1998) concluye, basándose en la gramática del inglés de Quirk et al. (1985: 1076), que las prótasis concesivas que modifican un evento (o "EdC") siempre siguen a la apódosis. En primer lugar notamos que en casi todos los usos de aunque - no sólo en nuestro corpus pero también en el corpus CREA - las dos cláusulas se dividen entre sí por una coma, lo cual simplemente se debe al hecho de que las reglas de puntuación para las concesivas españolas son menos estrictas que las del inglés (RAE 2010: 336-338), en el que la hipótesis está basada ${ }^{11}$. En segundo lugar, vimos que en nuestro corpus el orden mutuo de los componentes en la oración concesiva descriptiva no depende de su semántica, sino de aspectos discursivos, tales como el papel del contenido dentro del contexto inmediato (véase también Flamenco García 1999: 3815-3816). Consideremos un ejemplo más de una oración concesiva eventiva:

\footnotetext{
${ }^{10}$ Nótese que este uso del concepto de restrictividad es diferente del de Flamenco García, quien se refiere al aunque concesivo como "aunque restrictivo" (1999: 3819-3820) para distinguirlo del uso adversativo de aunque.

${ }^{11}$ Además de remitir a la gramática inglesa de Quirk et al. (1985: 1070-1077) Crevels (1998: 136) cita a Dik et al. (1990), quienes también toman el inglés como punto de salida.
} 
29. Por el camino, aunque la peluquería está cerca, ha tenido tiempo para ver un puesto de periódicos. Desde las portadas de todos los semanarios ilustrados, las veinteañeras del mundo entero, las que estaban naciendo o gestándose en Turín, la Unión Soviética, Oslo o Miami cuando ella tenía, a su vez, veinte años y cantaba canciones que ahora vuelve a traer el vaivén de la moda, la asaetan burlonamente con los ojos lánguidos o sonrientes y sus pelos lisos y largos, con moños, con trenzas, con pelucas, con tirabuzones. (Martín Gaite)

En este caso la apódosis funciona como enlace para el contenido que sigue, y la inversión del orden de las dos cláusulas sería, aunque gramatical, pragmáticamente infeliz. El caso es similar en los ejemplos de modificación de EEdCC (23)-(25) citados arriba, en los que la prótasis también precede a la apódosis. La oración en (23) aparece en la primera página de un cuento, en que se introduce a los personajes; la estructura me llamaba Zaratustra con la cual termina la oración, es paralela a la estructura con la que termina la oración anterior se llamaba Ermelinda. En (24), el malestar del narrador en primera persona es el tema del párrafo, que precede a la oración citada y sigue después de ésta; ya que el contenido de la apódosis continúa este tema, se presenta al final. En la oración en (25), el contenido de la prótasis es temático, y el de la apódosis es nuevo, y presenta un evento que forma parte del argumento de la novela. Estos cuatro ejemplos demuestran que el orden de prótasis y apódosis depende de las propiedades del contexto más amplio, o bien de la estructura del discurso. Obviamente, esto no vale solamente para la modificación concesiva de EEdCC, sino también para la de contenidos proposicionales.

Pasemos ahora a la estructura semántica de los contenidos proposicionales y los EEdCC visto desde la perspectiva de la gramática discursivo-funcional. La semántica forma parte del así llamado nivel representacional de la teoría, que junto al nivel interpersonal, mencionado en la introducción a este estudio, forma parte del subcomponente de formulación de la gramática. Lo que queda formulado en este subcomponente forma la entrada al subcomponente de codificación morfosintáctica y fonológica, del cual no nos ocuparemos aquí. La estructura básica del contenido proposicional se ve en (30a) y la del EdC en (30b):

\footnotetext{
30. a. (operador $\left.\mathrm{p}_{\mathrm{i}}:\left[\left(\mathrm{e}_{\mathrm{i}}\right)\left(\mathrm{e}_{\mathrm{j}}\right) \ldots . .\left(\mathrm{e}^{\mathrm{n}}\right)\right]:\left(\mathrm{p}_{\mathrm{j}}: \text { modificador }\right)_{\text {Función }}\right)$
}

b. (operador $\left.\mathrm{e}_{\mathrm{i}}:\left[\left(\mathrm{fc}_{\mathrm{i}}\right)\left(\mathrm{fc}_{\mathrm{j}}\right) \ldots .\left(\mathrm{fc}^{\mathrm{n}}\right)\right]:\left(\mathrm{e}_{\mathrm{j}} \text { : modificador }\right)_{\text {Función }}\right)$

En esta representación, se ve en primer lugar que las estructuras son completamente paralelas: los contenidos proposicionales (p) pueden tener uno o más EEdCC (e) dentro de su alcance y los EEdCC pueden tener una o más "propiedades configuracionales" (fc) dentro de su alcance. Una propiedad configuracional consta básicamente del predicado con sus argumentos. A diferencia de los EEdCC y los contenidos proposicionales, las propiedades configuracionales no tienen correspondencia ontológica con eventos o hechos, sino que representan la estructura temporal interna de un evento, que puede ser modificada por la operación de ciertos aspectos verbales, como por ejemplo los aspectos perfectivo/imperfectivo (cf. e.g. Comrie 1976: 3-4). Los operadores y modificadores en (30) pueden expresar contenidos similares entre sí, siendo la diferencia que los operadores conciernen a elementos gramaticales mientras que los modificadores conciernen a expresiones léxicas. Las funciones expresan las relaciones semánticas entre las entidades menores y las entidades mayores de las que forman parte. Cabe observar además que la representación en el nivel representacional no refleja el orden linear del modificador con respecto al elemento modificado. Por convención los modificadores siguen al elemento modificado en las representaciones.

La gramática discursivo-funcional puede servir de instrumento para dar una idea de la estructura semántica de la modificación concesiva descriptiva. Empecemos con la estructura de la modificación de los EEdCC. 
Para evitar complicaciones innecesarias simplifiquemos el ejemplo (23) como (23b). Entonces (23c) es una representación informal ${ }^{12}$ de la relación que existe entre la prótasis concesiva y la apódosis. La estructura interna de los dos EEdCC queda sin analizar, lo cual se indica por las barras horizontales que encierran los textos.

23. b. Aunque mi nombre es Marcos, Don Fortunato me llamaba Zaratustra.

c. (past $\mathrm{e}_{\mathrm{i}}$ :-Don Fortunato me llamaba Zaratustra-: (pres $\mathrm{e}_{\mathrm{j}}$ :-mi nombre es Marcos-) ${ }_{\text {Conc }}$ )

En esta representación, se ve que el EdC (o el "evento") $\left(\mathrm{e}_{\mathrm{i}}\right)$ se refiere a la cláusula principal, en la cual el EdC concesivo $\left(\mathrm{e}_{\mathrm{j}}\right)$ está incrustado, lo cual se representa a través de ":" el principal y las paréntesis internas que encierran el incrustado. Ambos EEdCC llevan marcas de tiempo: $\left(\mathrm{e}_{\mathrm{i}}\right)$ lleva operadores de tiempo pasado (past) y $\left(e_{j}\right)$ de presente (pres). La concesividad se representa por la función semántica en $\left(\mathrm{e}_{\mathrm{j}}\right)$, indicando la relación que existe entre el EdC subordinado y el principal $\left(\mathrm{e}_{\mathrm{i}}\right)$. Como las funciones respectan a elementos gramaticales, la función concesiva llegará a expresarse en el subcomponente de codificación por medio de la conjunción concesiva aunque, por ser la conjunción concesiva más gramaticalizada. Recuérdese que la representación semántica no refleja el orden mutuo de las cláusulas. Y como vimos en lo anterior, el orden mutuo de los dos EEdCC es independiente de la relación semántica expresada, y depende más bien de la estructura del texto o discurso

En el caso de la modificación proposicional, la representación es análoga. La oración en (26b) es una versión simplificada de la original. La representación informal correspondiente se encuentra en (26c):

26. b. Supongo que bromeas, aunque en tus palabras subyace un matiz de reproche c. ( $\mathrm{p}_{\mathrm{i}}$ : - supongo que bromeas-: ( $\mathrm{p}_{\mathrm{j}}$ : - en tus palabras subyace un matiz de reproche- $\left.)_{\text {Conc }}\right)$

Las estructuras de las concesivas descriptivas son muy similares, ya que se trata de EEdCC o proposiciones subordinadas en los dos casos. Como veremos ahora mismo, las concesivas interpersonales son de índole muy diferente.

\subsubsection{Las concesivas interpersonales}

Las concesivas interpersonales suelen constituir un comentario del hablante/escritor sobre su propia enunciación, pues lo que se expresa en la prótasis concesiva no está relacionado con el contenido de la apódosis, sino con las circunstancias comunicativas. Consideremos algunos ejemplos:

31. Se casará. Ahora ya no tiene excusa; ya lo verás. La que de todas sale ganando es su madre, que se libra de uno. Y de los peores [hijos], aunque no hay ninguno bueno en esa casa. (García Hortelano)

32. Leía, leía mucho. Julio Verne me apasionó. [...] También descubrí a Walter Scott y a Dickens, aunque este último me molestaba porque se movía por compasión. (Gironella)

33. Yo puedo decirle simplemente "te quiero", lo que no es verdad; o "quiero demostrar, a ti y a mí, que soy un hombre", y ésta es una gran verdad, pero demasiado evocador del ridículo fiasco primero; o "quiero acostarme contigo", exabrupto brutal para una señora de alta clase; o bien, al estilo de ahora, "vamos a hacer el amor", aunque esta expresión me repugna, siempre me ha parecido absurda: el amor no se hace, no se fabrica como el chocolate, el jabón o la gasolina, por un fugaz acto de ayuntamiento físico; (Soriano)

\footnotetext{
${ }^{12}$ Puesto que no nos ocupamos aquí de detalles técnicos de la teoría discursivo-funcional, todas las representaciones son informales y simplificadas, especificándose sólo los detalles imprescindibles en cada caso. Para más información sobre la teoría remitimos a los lectores a Hengeveld y Mackenzie (2008) para una versión completa, a Hengeveld y Mackenzie (2011) para una versión resumida y a Keizer (2015) para una versión didáctica.
} 
34. Lavar y marcar; pero no sé si cortarme también un poco. No mucho, como le cortaron el otro día a la señora de Soriano. ¿sabe cómo le digo?, así las puntas de delante un poco más largo, pero que quede liso, aunque no sé qué tal me estaría a mí..., es que no sé qué hacerme con el pelo, Carmen, le digo la verdad. (Martín Gaite)

Lo que tienen en común estos cuatro casos es que siempre se trata de algún tipo de autocorrección por parte del hablante. En los ejemplos (33) y (34) esta autocorrección tiene referencia directa a lo dicho anteriormente, mientras que en (31) y (32) la concesiva funciona para impedir posibles inferencias por parte del interlocutor (véase p. ej. Günthner 2000: 448): en el ejemplo (31) se anula la posible inferencia de haber hijos buenos y en (32), descubrir a Dickens, se impide que el lector infiera una evaluación positiva de Dickens por el narrador interno.

Otra propiedad que tienen en común los ejemplos (31) y (32) es el alcance de la prótasis: en ambos casos la prótasis sirve para comentar los actos discursivos que la preceden, $Y$ uno de los peores y También descubrí a Walter Scott y a Dickens. En (33), el alcance de la prótasis es incluso más reducida, ya que comenta la expresión hacer el amor, es decir que tiene una función claramente metalingüística. Estos tres ejemplos ilustran el tipo ilocutivo de las concesivas interpersonales (Crevels 1998: 137-139), que ya mencionamos en la introducción a nuestro trabajo. Desde nuestra perspectiva discursivo-funcional nos referiremos a este tipo como "concesiva de acto discursivo", siendo un "acto discursivo" la unidad mínima de actuación comunicativa. Tal unidad mínima de actuación comunicativa no forma necesariamente una contribución independiente a la comunicación, tal como se aprecia sobre todo en $Y$ de los peores del ejemplo $(31)^{13}$. El alcance de la concesiva es bastante más amplio en el ejemplo (34), porque la hablante usa la expresión concesiva para revisar o mitigar todo lo que ha dicho anteriormente a su interlocutora, la peluquera Carmen. Contiene un total de siete actos discursivos:

34. a. $A_{1}$ : Lavar y marcar $A_{2}$ : pero no sé si cortarme también un poco $A_{3}$ : No mucho, $A_{4}$ : como le cortaron el otro día a la señora de Soriano $\mathrm{A}_{5}$ : ¿ sabe cómo le digo?, $\mathrm{A}_{6}$ : así las puntas de delante un poco más largo, $\mathrm{A}_{7}$ :pero que quede liso

Como se ve, estos actos discursivos forman una unidad temática, lo cual es una de las propiedades características de una entidad mayor discursiva, el así llamado move 'movimiento', que puede consistir en uno o más actos discursivos y que sí forma una contribución independiente a la comunicación (Kroon 1997: 20) ${ }^{14}$. Siguiendo a Garcia (2010: 150-167), llamaremos este tipo "concesivas de movimiento" ("concesivas textuales" en Crevels 1998: 139).

Para indagar más sobre la estructura distinta de los dos tipos, consideremos algunos ejemplos más. El ejemplo (35) ilustra la concesiva de acto discursivo, que esta vez no es autocorrección:

35. [conversación con una mujer de 52 años] bueno / aunque la jubilación está lejos /// e... ¿qué piensas hacer cuando te jubiles // o cómo... organizarás tu jubilación? (Gómez Molina 2001: 139)

El caso de (35) es similar al ejemplo (7) que citamos en la introducción, con la diferencia de que en (7), la prótasis sigue a la apódosis, mientras que aquí el orden mutuo es al revés. Igual como en el ejemplo (7), el contenido de la prótasis en (35) especifica la pertenencia (o "felicidad") del acto discursivo contenido en la apódosis, indicando que el hablante está consciente de que éste no es del todo pertinente. Como tal, sirve para mitigar este último acto discursivo.

\footnotetext{
13 "Acts (or discourse acts, as I prefer to call them) can be defined as the smallest identifyable units of communicative behaviour. In contrast to higher order units called moves they do not necessarily further the communication in terms of approaching a conversational goal." (Kroon 1997: 20).

14 "The higher-rank unit called move is defined as the minimal free unit of discours that is able to enter into an exchange structure. Unlike the act, a move is defined not only in terms of communicative unity but also in terms of thematic unity."
} 
Es decir que aquí sigue habiendo una clara relación con la semántica concesiva: el acto se realiza pese a que las condiciones contextuales no favorecen su realización. Dicho de otra manera: dadas la situación y/o el contexto $p$, normalmente no se realizaría $q$.

Veamos ahora un ejemplo de movimiento:

36. Debo reconocer que precisamente por llevar la contraria, desde chico he leído a escondidas muchos libros prohibidos, que antes me parecían los mejores; [...] Pero ahora ya no pienso que la prohibición de cualquier cosa sea garantía cierta de su buena calidad: ahora soy más ecléctico -es decir, soy posmoderno-, aunque mejor diría que cada vez soy menos capaz de fervores exaltados y que buena parte de los libros devorados me pesan en la mente como pesan en el estómago ciertas comidas indigestas. (Soriano)

En este ejemplo aunque introduce un movimiento nuevo, que sigue, como rectificación del movimiento anterior Pero ahora ya no pienso que la prohibición de cualquier cosa sea garantía cierta de su buena calidad: ahora soy más ecléctico - es decir, soy posmoderno-, que ya contiene una reformulación. A diferencia de las prótasis de acto discursivo (o "ilocutivas"), las prótasis de movimiento (o "textuales") siempre consisten en rectificaciones. Como tal, nunca pueden preceder a la apódosis, porque lógicamente una corrección sólo puede seguir a lo que hace falta corregir ${ }^{15}$. Otra diferencia es que en las concesivas de movimiento es más difícil establecer una relación clara con la concesión. Lo que expresa aunque en este tipo de casos es un significado de 'disonancia e incompatibilidad' (Günthner 2000: 458) por lo cual la delimitación entre la concesión y la adversatividad se difumina en el uso de movimiento (cf. también König 1994: 681). Sin embargo, coincidimos con Flamenco García (1999: 3820), quien observa al respecto que aunque en este tipo de contextos expresa una oposición más atenuada frente a pero.

La gramática discursivo-funcional nos permite sistematizar la diferencia entre los dos tipos concesivos interpersonales. El nivel interpersonal de la GDF se ocupa de la relación entre los elementos de la enunciación: (i) los actos discusivos (A) que, entre otras, incluyen una ilocución y un contenido comunicado, y (ii) los movimientos (M), unidades de enunciación temáticamente coherentes consistentes de uno o más actos discursivos. Así que una versión simplificada de (35) debería ser representada como (35a):

35. a. $\left(M_{i}\left(A_{i}-\text { la jubilación está lejos- }\right)_{\text {Conc }}\left(A_{j}:-¿\right.\right.$ ¿qué piensas hacer cuando te jubiles?-))

Como se ve aquí, los dos actos juntos forman un movimiento. Nótese que a diferencia del nivel representacional, el orden mutuo de los componentes en el nivel interpersonal refleja el orden cronológico de enunciación. Así que en una representación del ejemplo (7), la prótasis estaría en el segundo lugar. Siendo altamente gramaticalizada, la conjunción aunque se expresa otra vez como una función que refleja la relación del acto discursivo dependiente con el acto principal $\left(\mathrm{A}_{\mathrm{j}}\right)$. Pero esta vez la función no expresa una relación semántica sino una relación retórica. Otra diferencia crucial entre la concesiva interpersonal representada aquí y la concesiva descriptiva, representada por ejemplo en (23c), es que aquí no hay incrustación: los dos actos discursivos están interaccionalmente relacionados en el sentido de que el acto concesivo es dependiente del acto discursivo no marcado, pero no hay dependencia en un sentido semántico-sintáctico.

La representación de los movimientos en una versión simplificada de (36) en (36a) tiene mucho en común con la del acto discursivo concesivo:

36. a. $\left(\mathrm{M}_{\mathrm{i}}\right.$ :-pero ahora ya no pienso que $[\ldots]$ soy posmoderno-) $\left(\mathrm{M}_{\mathrm{j}} \text { :-mejor diría que }[\ldots] \text { indigestas- }\right)_{\text {Rect }}$

\footnotetext{
${ }^{15}$ Para más detalles sobre la prótasis concesiva de movimiento véase Parra (2016).
} 
Una diferencia importante es la función retórica del movimiento dependiente. La etiqueta de "rectificación" (Rect), basada en König (1994: 681), da cuenta del hecho de que se ha perdido la relación con la concesión propiamente dicha y también de la irreversibilidad del orden mutuo entre el movimiento principal y el movimiento dependiente ${ }^{16}$.

\subsection{Resumen}

En esta sección nos hemos ocupado de las características de las oraciones concesivas propias con la conjunción aunque. Hemos demostrado (i) que la concesión se distingue de la adversatividad por ser un caso de subordinación adverbial, donde la unión de las dos cláusulas se opone a una relación causal presupuesta; (ii) que, de modo general, el uso del subjuntivo en la prótasis sirve para indicar que el hablante presupone el conocimiento del contenido de ésta en el oyente, lo cual, en términos discursivo-funcionales se puede explicar recurriendo al componente contextual del modelo comunicativo; y (iii) cómo se distinguen entre sí los usos descriptivos e interpersonales, aspecto que hemos indagado en más detalle. Dentro de los usos descriptivos hay que distinguir dos estratos de incidencia de la prótasis concesiva, el eventivo y el proposicional, distinción que se puede comprobar por la inserción de un adverbio epistémico en la apódosis. Las concesivas interpersonales también vienen en dos subtipos: en el primero, la prótasis concesiva modifica un acto discursivo único y en el segundo modifica toda una serie de actos discursivos, es decir, un movimiento. Las diferencias entre estos tipos de concesión las hemos explicado en términos discursivo-funcionales: las concesivas descriptivas pertenecen al nivel representacional o semántico de la gramática, donde funcionan como modificadores léxicos de EEdCC (e) o de contenidos proposicionales $(\mathrm{p})$; las prótasis concesivas interpersonales actúan en el nivel interpersonal como actos discursivos o movimientos dependientes que modifican actos discursivos o movimientos principales. En el caso de los actos discursivos, tienen función retórica de concesión, pero en los movimientos tienen función retórica de corrección, porque en este caso ya no se expresa concesión propiamente dicha.

\section{Oraciones concesivo-condicionales con aunque}

Como ya mencionamos en la sección 2, las oraciones concesivo-condicionales con aunque pertenecen al grupo escalar y comparten propiedades tanto con las condicionales como con las concesivas: las prótasis concesivo-condicionales son hipotéticas igual que las prótasis condicionales, y el contenido de las prótasis crea una expectativa que es contradicha por el contenido de la apódosis, igual que en las oraciones concesivas. Aquí nos ocuparemos primero de las propiedades condicionales típicas de las concesivo-condicionales escalares, (4.1.) y después de sus propiedades concesivas, enfocándonos particularmente en las que son distintas de las concesivas (4.2.).

\subsection{Las propiedades condicionales}

Para empezar, recordemos que una de las etiquetas de la concesivo-condicional es la de la "condicional de irrelevancia", porque pese al carácter hipotético de la prótasis, la apódosis siempre es factual:

37. voy a transformar mi tienda [...] aunque me cueste un poco más caro (Moreno Fernández et al 2004: 395)

\footnotetext{
${ }^{16}$ Lo que puede llamar la atención en esta representación es que no hay estrato superior al movimiento que tenga a los dos movimientos dentro de su alcance en analogía a la relación entre los actos y los movimientos representada en (35a). Para tal estrato superior, Kroon (1997: 20-21), por ejemplo, propone "exchange" para la interacción dialógica y "extended monologue" para la narrativa, y Stassi-Sé (2012: 171-176) propone "discurso" para las dos cosas. Sin embargo, Hengeveld y Mackenzie (2008: 28) aclaran que, no obstante su nombre, la GDF no es una gramática del discurso, sino que se ocupa de las propiedades lingüísticas del acto discursivo como unidad mínima comunicativa.
} 
En (37) la transformación de la tienda va a ser realizada independientemente de si el precio es alto o bajo. La primera propiedad condicional de las concesivo-condicionales escalares que queremos discutir aquí es la de 'especificar una serie de valores posibles aseverando una relación condicional para un valor extremo en una escala imaginaria ${ }^{17}$. Dicho de otro modo: lo que viene descrito en la apódosis por implicación también vale para condiciones menos extremas, como se puede apreciar en (37) y en el siguiente ejemplo:

38. [de una carta de amor] Rocío es usted, únicamente usted, y aunque en su tierra existan cientos de Rocíos, para mí, desde hace tres meses, no hay más que una. (Delibes)

En el ejemplo (37), la prótasis concesivo-condicional implica que el hablante también va a transformar su tienda si le resulta menos caro o incluso barato. Y en (38) la prótasis expresa una condición extremadamente improbable, y queda claro que la unicidad de la destinataria también quedaría en pie si hubiera sólo decenas, cinco o incluso ninguna otra Rocío en su tierra.

Al mismo tiempo este ejemplo aclara la función discursivo-pragmática de las concesivocondicionales, que consiste en enfatizar el contenido que se comunica. Una representación de (38) sería:

38. a. ( $\mathrm{p}_{\mathrm{i}}$ : -para mí [...] no hay más que una-: (hyp $\mathrm{p}_{\mathrm{j}}$ : -en su tierra existen cientos de Rocíos-)

Mientras que la concesión es una relación entre dos unidades lingüísticas, y por tanto en términos discursivo-funcionales se realiza como función semántica (Conc), la hipótesis es una propiedad de una sola unidad lingüística, y por eso se expresa como operador de ésta (hyp). Este operador opera siempre sobre una proposición, ya que las hipótesis no se pueden colocar ni en el tiempo ni en el espacio sino que son constructos mentales. Lo que tienen en común las funciones y los operadores es que tienen expresión gramatical. En este caso el efecto combinado de la función concesiva y del operador hipotético resulta en la combinación de la conjunción aunque con el modo subjuntivo en el verbo.

La segunda propiedad típicamente condicional de las concesivo-condicionales es la de poder expresar tanto condiciones potenciales, como las que vimos en los ejemplos presentados hasta ahora, como condiciones irreales. Considérense los siguientes ejemplos:

39. [Un guerrillero se esconde en un pajar]

Pero ni aunque cosieran el pajar de extremo a extremo con palos y guadañas podrían encontrarme. (Llamazares)

40. [En un selecto restaurante exclusivamente femenino la comida resulta ser malísima]

Supe en seguida que, aunque hubieran admitido caballeros, aquél no era un buen lugar para invitar al subinspector. (CREA ficción, A. Giménez Barlett, Serpientes en el paraíso, 2002)

Pese a la aparente diferencia en la realización temporal entre los dos ejemplos, ambos ejemplos son igualmente contrafactuales: la diferencia entre imperfecto de subjuntivo en (39) y el pluscuamperfecto de subjuntivo en (40) se debe exclusivamente a la consecutio temporum, es decir, a la adaptación del tiempo en la cláusula subordinada al tiempo del contexto, este último siendo presente en (39) y pasado en (40). Según Harris (1988: 73) tanto las cláusulas condicionales como las concesivo-condicionales dejan de ser hipotéticas cuando el contenido es contrafactual. Sin embargo, en nuestra opinión la naturaleza hipotética de la prótasis condicional o concesivocondicional no depende de la realidad o irrealidad del contenido expresado.

\footnotetext{
17 "a series of antecedents is specified by asserting a conditional relationship for an extreme (unlikely) value on a scale" (König 1985: 4); véase también König (1994: 640).
} 
Siguiendo a Hengeveld (2014: 1197), consideramos la expresión de irrealidad como modificación de los EEdCC mientras que el operador hipotético tiene el operador pasado, al cual se debe la irrealidad, dentro de su alcance. Así que (40) se debería analizar del modo siguiente ${ }^{18}$ :

40. a. ( $\mathrm{p}_{\mathrm{i}}$ : -aquél no era un buen lugar para invitar al subinspector-: (hyp $\mathrm{p}_{\mathrm{j}}$ : [past $\mathrm{e}_{\mathrm{i}}$ :-admit- caballeros-]) ${ }_{\text {Conc }}$ )

El efecto combinado del operador hipotético (hyp) en el estrato de la proposición con el operador pasado (past) en el del EdC se expresaría como imperfecto de subjuntivo. La consecutio temporum no se representa aquí puesto que es un efecto de la aplicación de las reglas morfosintácticas, que quedan fuera de consideración en este contexto. Se ve en (40a) que, en principio, la relación semántica entre la proposición incrustada y la principal sigue siendo la misma como en (38a), igual que la propiedad hipotética. La diferencia entre la concesivo-condicional potencial y la contrafactual reside en la realidad o irrealidad del EdC.

\subsection{Las propiedades concesivas}

A pesar de que en general las propiedades semánticas y pragmáticas de las concesivas con aunque también son aplicables a las concesivo-condicionales, existen diferencias notables con respecto al alcance entre los dos tipos, tanto en el uso descriptivo como en el interpersonal.

En lo que concierne al uso descriptivo, el alcance de la prótasis concesivo-condicional puede ser el contenido proposicional como en el ejemplo (40) discutido en detalle en la sección 4.1., pero también puede ser un EdC, como (37), que repetimos aquí por conveniencia:

37. voy a transformar mi tienda [...] aunque me cueste un poco más caro (Moreno Fernández et al. 2004: 395)

La diferencia se ve en la siguiente representación discursivo-funcional:

37. a. (prosp $\mathrm{e}_{\mathrm{i}}$ :-transformar mi tienda $\left(\mathrm{x}_{\mathrm{i}}\right)$-: (hyp $\mathrm{p}_{\mathrm{j}}$ : -me cuesta un poco más caro-) $\left.)_{\text {Conc }}\right)$

El operador prospectivo delante de la variable eventiva $\left(\mathrm{e}_{\mathrm{i}}\right)$, se refiere a la perífrasis ir $a \mathrm{y}\left(\mathrm{x}_{\mathrm{i}}\right)$ es la variable que se refiere al agente implícito.

Una categoría específica de las cláusulas concesivo-condicionales son las del tipo aunque (solo) sea, que expresan una "condición mínima" como apuntan Garachana Camarero (1999: 198) y RAE (2009: 3604). En estos casos la prótasis no establece un contraste con una apódosis sino que la cláusula concesivo-condicional sirve exclusivamente para describir una propiedad - en términos predicativos o adverbiales - de un elemento nominal o verbal del resto del enunciado. Considérense los siguientes ejemplos:

41. Lo invitaré, aunque solo sea por cortesía. (RAE 2009: 3604)

42. Así, si en algún punto del borde del área pretendidamente cerrada existe una hendidura, aunque sólo sea de un píxel, el color escapará de sus límites pudiendo llegar a llenar toda la pantalla y estropear todo el trabajo realizado hasta ese momento. (CREA informática, M. Pardo Niebla, Windows 2000. Guía práctica para usuarios, 2000)

43. Si el embarazo se considera de alto riesgo y existe amenaza de aborto en el primer trimestre o de parto prematuro en el segundo y el tercero, la mujer no debe moverse de su ciudad y consultar con su médico cualquier traslado, aunque sea corto. (CREA medicina, E. García del Real, Nueva guía de ginecología, 1999)

\footnotetext{
${ }^{18}$ Recuérdese que todas las representaciones no contienen más de la información estrictamente necesaria. Mientras que en (37a) no especificamos el estrato del EdC, lo representamos aquí por ser imprescindible para nuestro propósito. La representación del tiempo pasado se da sólo en la prótasis, mientras que en la apódosis queda implicado en la proposición sin analizar.
} 
Esta propiedad mínima hipotética es el valor extremo que es característico de las concesivocondicionales, tal valor mínimo siendo atípico de la propiedad designada por el elemento modificado. Es en este sentido que la prótasis concesivo-condicional expresa un contraste con lo que se esperaría "bajo circunstancias normales". Nótese que la prótasis está pospuesta al elemento que modifica, a saber, invitar (con sus argumentos) en (41), una hendidura en (42) y cualquier traslado en (43): invitar a alguien sólo por cortesía es una invitación "minima" en el sentido de hacerse de mala gana (41), una hendidura de un píxel es una hendidura mínima (42) y un traslado corto es un traslado mínimo (43). Una estructura informal de (43) debería ser algo como:

$$
\text { 43. a. ( } \left.\left.\mathrm{e}_{\mathrm{i}}:- \text { cualquier traslado -: (hyp } \mathrm{p}_{\mathrm{i}}:- \text { es corto }-\right)_{\text {Conc }}\right)
$$

Por ser evento, cualquier traslado se representa como EdC $\left(\mathrm{e}_{\mathrm{i}}\right)$. Como siempre la combinación de la función concesiva con el operador hipotético resulta en aunque con el subjuntivo en la expresión morfosintáctica.

Por lo que atañe a las concesivo-condicionales interpersonales, no existe ningún caso en nuestro corpus. Por lo que sabemos, las concesivo-condicionales interpersonales con aunque están limitadas a la modificación de actos discursivos. Considérense los siguientes ejemplos:

44. $\mathrm{Y}$ es que, aunque parezca mentira, siglo y medio después de la publicación de El origen de las especies, no son pocos quienes siguen creyendo que el hombre llegó al mundo en el 4004 a.C.

(CREA antropología, B. Cardeñosa, El código secreto, 2001)

45. Aunque te parezca una aberración, hoy día presumir de anticlerical es presumir de retrógrado ... (CREA ficción, M. Salisachs, La gangrena, 1975)

Lo interesante en este tipo de casos es que la concesión es una relación que se establece en el nivel interpersonal, mientras que la hipótesis sigue siendo una propiedad proposicional. Es decir que para dar cuenta de este tipo de casos en la gramática discursivo-funcional hay que tomar en cuenta tanto el nivel interpersonal (NI) como el representacional (NR). Así que (45) se debería representar como:

45. a. NI: $\left(\mathrm{A}_{\mathrm{i}} \text { :-te parece una aberración- }\right)_{\mathrm{Conc}}$ NR: (hyp $p_{i}$ )
( $\mathrm{A}_{\mathrm{j}}$ :-hoy día presumir de anticlerical es presumir de retrógrado-)

$\left(\mathrm{p}_{\mathrm{j}}\right)$

Como en las oraciones concesivo-condicionales discutidas hasta ahora, la combinación de la función concesiva y el operador hipotético desencadenan la codificación por medio de la conjunción altamente gramaticalizada aunque y el modo subjuntivo. Igual que en las concesivas interpersonales presentadas en la sección 3.3.2., no hay subordinación aquí, es decir que la prótasis no queda incrustada dentro de la oración; la dependencia entre las dos cláusulas es de índole interpersonal.

\section{Conclusión}

En el presente trabajo hemos dado una visión panorámica de los usos posibles de la conjunción aunque tanto en las oraciones concesivas propias como en las concesivo-condicionales, y tanto en los uso descriptivos como en los interpersonales. Esperamos haber demostrado en este trabajo que es posible explicar hasta el nivel del acto discursivo, como unidad mínima de actuación comunicativa, todo tipo de uso de aunque haciendo uso de los instrumentos que nos ofrece el modelo discursivo-funcional. Lo que queda por explicar, tal vez dentro del marco de un estudio textual o estilístico, es el orden mutuo de la prótasis y la apódosis en el uso descriptivo, que no se puede aclarar por medio de un modelo gramatical. 


\section{Referencias bibliográficas}

\section{Estudios}

Anscombre, Jean-Claude y Oswald Ducrot. 1977. Deux mais en français?, Lingua, 43,1: 23-40.

Bülow, Lars y Christoph Schamberger. 2013. Die Logik indikativischer wenn-Sätze: eine philosophisch-linguistische Studie zum konditionalen und konzessiven Gebrauch des Subjunktors wenn, Zeitschrift für germanistische Linguistik, 41, 2: 277-298.

Comrie, Bernard. 1976. Aspect, Cambridge, Cambridge University Press.

Crevels, Mily. 1998. Concession in Spanish, en Mike Hannay y A. Machtelt Bolkestein (eds.), Functional grammar and verbal interaction, Amsterdam, Benjamins: 129-148.

Crevels, Mily. 2000. Concessives on different semantic levels: a typological perspective, en Elizabeth Couper-Kuhlen y Bernd Kortmann (eds.), Cause, condition, concession, contrast, Berlin, Mouton de Gruyter: 313-339.

Dik, Simon C., Kees Hengeveld, Elseline Vester y Co Vet. 1990. The hierarchical structure of the clause and the typology of adverbial satellites, en Jan Nuyts, A. Machtelt Bolkestein y Co Vet (eds.), Layers and levels of representation in language theory: a functional view, Amsterdam, John Benjamins: 25-70.

Flamenco García, Luis. 1999. Las construcciones concesivas y adversativas, en Ignacio Bosque y Violeta Demonte (directores), Gramática descriptiva de la lengua española, Vol. 3, Madrid, Espasa: 3805-3878.

Garachana Camarero, Mar. 1999. Valores discursivos de las oraciones concesivas, Lingüística española actual, 21, 2: 189-205.

Garcia, Talita Storti. 2010. As relações concessivas no português falado sob a perspectiva da Gramática Discursivo-Funcional. Tese de doutorado, Universidade Estadual Paulista. São José de Rio Preto. Disponible en: http://repositorio.unesp.br/handle/11449/100114.

Günthner, Susanne. 2000. From concessive connector to discourse marker: the use of obwohl in everyday German interaction, en Elizabeth Couper-Kuhlen y Bernd Kortmann (eds.), Cause, condition, concession, contrast, Berlin, Mouton de Gruyter: 439-468.

Harris, Martin. 1988. Concessive clauses in English and Romance, en John Haiman y Sandra A. Thompson (eds.), Clause combining in grammar and discourse, Amsterdam, John Benjamins: 71-99.

Hengeveld, Kees. 2014. Mood and modality, en Geert Booij, Christian Lehmann y Joachim Mugdan (eds.), Morphology: a handbook of inflection and word formation, Vol. 2. Berlin, Mouton de Gruyter: 1190-1202.

Hengeveld, Kees y J. Lachlan Mackenzie. 2008. Functional discourse grammar: a typologicallybased theory of language structure, Oxford, Oxford University Press.

Hengeveld, Kees y J. Lachlan Mackenzie. 2011. La gramática discursivo-funcional (traducción española por Daniel García Velasco), Moenia, 17: 5-45.

Haspelmath, Martin y Ekkehard König. 1998. Concessive conditionals in the languages of Europe, en Johan van der Auwera (ed.), Adverbial constructions in the languages of Europe, Berlin, Mouton de Gruyter: 563-640.

Keizer, Evelien. 2015. A functional discourse grammar for English, Oxford, Oxford University Press.

König, Ekkehard. 1985. On the history of concessive connectives in English: diachronic and synchronic evidence, Lingua, 66: 1-19.

König, Ekkehard. 1988. Concessive connectives and concessive sentences: cross-linguistic regularities and pragmatic principles, en John A. Hawkins (ed.), Explaining language universals, Oxford, Basil Blackwell: 145-166.

König, Ekkehard. 1994. Concessive clauses, en R. E. Asher (ed.), The encyclopaedia of language and linguistics, Vol. 2, Oxford, Pergamon Press: 679-681. 
Kroon, Caroline. 1997. Discourse markers, discourse structure and Functional Grammar, en John H. Connolly, Roel Vismans, Christopher S. Butler y Richard A. Gatward (eds.), Discourse and pragmatics in Functional Grammar, Berlin, Mouton de Gruyter: 17-32.

Lyons, John. 1977. Semantics. Cambridge, Cambridge University Press.

Neves, Maria Helena de Moura. 1999. As construções concessivas, en Maria Helena de Moura Neves (org.), Gramática do português falado. Campinas, Editora da Unicamp.

Parra, Beatriz Goaveia Garcia. 2016. Uma investigação discursivo-funcional das orações concessivas introduzidas por aunque em dados do espanhol peninsular. Dissertação de Mestrado, Universidade Estadual Paulista, São José de Rio Preto, Inédita.

Quirk, Randoph, Sidney Greenbaum, Geoffrey Leech y Jan Svartvik. 1985. A comprehensive grammar of the English language. London, Longman.

Real Academia Española y Asociación de Academias de la Lengua Española. 2009. Nueva gramática de la lengua española, 3 vols, Madrid, Espasa.

Real Academia Española y Asociación de Academias de la Lengua Española. 2010. Ortografía de la lengua española, Madrid [en línea]. Disponible en: www.rae.es [Acceso: 12/09/2015].

Rodríguez Rosique, Susana. 2012. From discourse to grammar: when Spanish incluso meets si conditional, Lingvisticae Investigationes, 35, 1: 94-119.

Stassi-Sé, Joceli C. 2012. Subordinação discursiva no português à luz da gramática discursivofuncional. Tese de Doutorado, Universidade Estadual Paulista, São José do Rio Preto. Disponible en: http://repositorio.unesp.br/handle/11449/100102.

Sweetser, Eve E. 1990. From etymology to pragmatics: metaphorical and cultural aspects of semantic structure, Cambridge, Cambridge University Press.

Vendler, Zeno. 1967. Facts and events, en Zeno Vendler, Linguistics in philosophy, Ithaca, NY, Cornell University Press: 122-146.

\section{Corpus literario}

Delibes, Miguel. 1983. Cartas de amor de un sexagenario voluptuoso (fragmento), Barcelona, Destinolibro: 62-92.

García Hortelano, Juan. 1987. Mucho cuento (fragmento), Madrid, Mondadori: 11-43.

Gironella, José María. 1983. Cita en el cementerio (fragmento), Barcelona, Planeta: 26-57.

Llamazares, Julio. 1985. Luna de lobos (fragmento), Barcelona, Seix Barral: 11-39.

Martín Gaite, Carmen. 1978. Cuentos completos (fragmento), Madrid, Alianza: 149-158.

Merino, José María. 1982. Cuentos del reino secreto, Madrid, Alfaguara: 143-159.

Ortiz, Lourdes. 1982. Pasajes y figuras en Ymelda Navajo (ed.) Doce cuentos de mujeres, Madrid, Alianza: 113-125.

Palomino, Ángel. 1985. El pecado de Paquita (fragmento), Barcelona, Planeta: 63-100.

Pombo, Álvaro. 1990. El pésame, en Juan Eslava Galán et al., El fin del milenio, Barcelona, Planeta: 75-105.

Puértolas, Soledad. 1990. Camino de Houmt Souk, en Juan Eslava Galán et al., El fin del milenio, Barcelona, Planeta: 109-136.

Rico Godoy, Carmen. 1990. Como ser una mujer y no morirse en el intento (fragmento), Madrid, Ediciones Temas de Hoy: 68-95.

Soriano, Elena. 1989. La vida pequeña (fragmento), Barcelona, Plaza y Janés: 109-138.

Tomeo, Javier. 1990. El artefacto, en Juan Eslava Galán et al., El fin del milenio, Barcelona, Planeta: 139-164.

Tusquets, Esther. 1979. El amor es un juego solitario (fragmento), Barcelona, Lumen: 20-32.

Vázquez Montalbán, Manuel. 1987. Historias de padres e hijos (fragmento), Barcelona, Planeta: $12-44$. 


\section{Corpus adicional}

Gómez Molina, José Ramón (coord.). 2001. El español hablado de Valencia : materiales para su estudio: (PRESEEA). 2, Nivel sociocultural medio, Valencia: Universitat de València.

Moreno Fernández, Francisco, Ana M. ${ }^{a}$ Cestero Mancera, Isabel Molina Martos y Florentino Paredes García. 2004. La lengua hablada en Alcalá de Henares: Corpus PRESEEA-ALCALÁ. II. Hablantes de instrucción media, Alcalá de Henares, Universidad de Alcalá.

Real Academia Española. Corpus de referencia del español actual. Madrid [en línea]. Disponible en <http://www.rae.es> [Acceso: 3 de noviembre de 2015] 